\title{
Search for very long-lived isomers in the hafnium-tungsten region
}

\author{
Zs. Podolyák, ${ }^{1}$ P. M. Walker, ${ }^{1}$ D. M. Cullen, ${ }^{2}$ G. D. Dracoulis, ${ }^{3}$ P. Fallon, ${ }^{4}$ B. Fairchild, ${ }^{4}$ K. Hauschild, ${ }^{5}$ A. O. Macchiavelli, ${ }^{4}$ \\ D. P. McNabb, ${ }^{5}$ A. Savelius, ${ }^{6}$ D. Ward, ${ }^{4}$ and C. Wheldon ${ }^{1}$ \\ ${ }^{1}$ Department of Physics, University of Surrey, Guildford GU2 7XH, United Kingdom \\ ${ }^{2}$ Department of Physics, Oliver Lodge Laboratory, University of Liverpool, Liverpool L69 7ZE, United Kingdom \\ ${ }^{3}$ Department of Nuclear Physics, Research School of Physical Sciences and Engineering, Australian National University, \\ Canberra ACT 0200, Australia \\ ${ }^{4}$ Lawrence Berkeley National Laboratory, Berkeley, California 94720 \\ ${ }^{5}$ Lawrence Livermore National Laboratory, Livermore, California 94550 \\ ${ }^{6}$ Department of Physics, University of Jyväskylä, Jyväskylä F-40351, Finland
}

(Received 25 August 1999; revised manuscript received 8 May 2000; published 3 August 2000)

\begin{abstract}
Targets of ${ }^{48} \mathrm{Ca}$ have been bombarded with ${ }^{134,136} \mathrm{Xe}$ beams as part of a search for long-lived, high- $K$ states in well-deformed nuclei with $A \approx 180$. Several known isomers were observed by off-line $\gamma$-ray detection, and limits have been established for high- $K$ isomer production in various isotopes of hafnium $(A=175-178)$, tantalum $(A=177-181)$, and tungsten $(A=177-181)$.
\end{abstract}

PACS number(s): 21.10.Tg, 27.70.+q

\section{INTRODUCTION}

The structure of nuclei in the Yb-Hf-W-Os $(Z=70-76)$ region is characterized by the presence of high-angularmomentum intrinsic, i.e., noncollective states. Such states are favored over collective excitations because the proton and neutron Fermi levels are among quasiparticle orbitals with large angular-momentum projections $(\Omega)$ on the symmetry axis, allowing states with large values of $K=\Sigma \Omega$ to be formed. These states may be long lived, with half-lives ranging from a few nanoseconds to several years, one of the most famous being the $K^{\pi}=16^{+}, 31 \mathrm{yr}$ isomer in ${ }^{178} \mathrm{Hf}$ [1].

There are several predictions concerning high- $K$ isomers in the $A \approx 180$ region [2-5], and some of these isomers are possibly very long lived. In ${ }^{178} \mathrm{~W}$ Jain et al. [3] predicted among others a low-lying eight-quasiparticle isomer with $K^{\pi}=30^{+}$which may decay to members of the $K^{\pi}=23^{+}$or $K^{\pi}=25^{+}$rotational bands. Due to a possible high multipolarity of its decay radiation, this state might be very long lived [6]. In ${ }^{180} \mathrm{Ta}$ several isomers in the spin region of 20-28 are predicted, although the highest spin identified multiquasiparticle state has a spin parity of $19^{(-)}[4,7]$. A $22^{-}$six-quasiparticle state may be expected to decay via an M3 transition to a $19^{-}$state, resulting in a possible very long-lived isomer [4]. The situation is very similar in ${ }^{177} \mathrm{Ta}$. The highest spin known multiquasiparticle state has spin parity of 49/2 $[8,9]$. Among the predicted higher spin isomeric states [8] there is one with a spin-parity of $67 / 2^{-}$, which may be expected to decay by an $E 3$ transition of $\sim 0.5 \mathrm{MeV}$, leading to a long lifetime. However, it is extremely difficult to predict lifetimes accurately, since they strongly depend on the deexcitation multipolarities, $K$-value changes and energies, and therefore on the fine details of the level schemes.

The goal of the present work is to search for high-spin isomers with half-lives longer than $1 \mathrm{~h}$ in the W-Ta-Hf nuclei. Since high angular momenta are needed the nuclei should ideally be populated in heavy-ion fusion-evaporation reactions, but to gain access to the most favored nuclei, such as those considered above, neutron-rich targets and beams are needed. One suitable combination of stable beams and targets is ${ }_{20}^{48} \mathrm{Ca}$ and each of the xenon isotopes: ${ }_{54}^{134} \mathrm{Xe}$ and ${ }_{54}^{136} \mathrm{Xe}$. In contrast to in-beam measurements, where rate limitations apply due to intense prompt radiations, increased sensitivity to long-lived isomers can be achieved by performing bombardments with high beam intensities followed by periods of off-line $\gamma$-ray counting.

The background to the motivation for such a study is that the discovery of new isomers may be used to test a number of nuclear structure phenomena such us configuration dependent pairing effects, Coriolis effects, shape coexistence, triaxiality, etc. [5]. For example, it is well known that the nuclear pairing force is responsible for the reduction of the moment of inertia well below that of a rigid body. In a $K$-isomeric state, the orbitals contributing to its configuration are blocked, therefore an increased moment of inertia is expected. The study of the several already identified high- $K$ isomers confirms the increase of the moment of inertia, but also suggest that its maximum value is $\approx 30 \%$ lower than that of a rigid body [10-12]. This value is reached in some ${ }^{178} \mathrm{~W}$ eight-quasiparticle states $[6,11]$ but more experimental data are needed.

This work is part of a wider research program to study high- $K$ isomers, with a range of lifetimes in the $A \approx 180$ region, using reactions with different population mechanisms, e.g., incomplete fusion [13], deep-inelastic [14], and fragmentation [15] reactions.

\section{EXPERIMENTAL MEASUREMENTS}

Isotopically enriched $(80 \%-90 \%){ }^{48} \mathrm{Ca}$-oxide targets of thickness $>10 \mathrm{mg} / \mathrm{cm}^{2}$ have been bombarded with $\approx 10$ particle-nA beams of ${ }^{134} \mathrm{Xe}$ and ${ }^{136} \mathrm{Xe}$. The beams were delivered by the 88-In. Cyclotron at the Lawrence Berkeley National Laboratory. The incident beam energy was 535 $\mathrm{MeV}$ in the case of ${ }^{134} \mathrm{Xe}$, and $535 \mathrm{MeV}$ and $550 \mathrm{MeV}$ in the case of ${ }^{136} \mathrm{Xe}$. The target consisted of ${ }^{48} \mathrm{Ca}$ oxide pressed into a tantalum mesh. The use of this chemical compound is 
justified by previous experience which showed that calcium oxide can withstand intense beams. The Coulomb barrier is at a higher laboratory energy for oxygen than for ${ }^{48} \mathrm{Ca}$, so that the reaction cross sections for xenon on oxygen are relatively small at the beam energies chosen. The irradiations were performed in a separate high-intensity beam line to avoid contamination of the measurement area.

The reaction conditions populate mainly ${ }^{177-181} \mathrm{~W}$ isotopes, after the evaporation from the compound nuclei of 3-5 neutrons, while ${ }^{177-181} \mathrm{Ta}$ and ${ }^{175-178} \mathrm{Hf}$ isotopes are also reached, with considerably lower cross sections, in $p x n$ and $\alpha x n$ channels, respectively. After irradiation, the target was mounted in the center of the $8 \pi \gamma$-ray detector array [16] consisting of 20 Compton-suppressed germanium detectors and a 70-element bismuth-germanate calorimeter. Events were written to tape when at least one Comptonsuppressed Ge detector and one BGO detector fired in coincidence (which improves the discrimination against background). Another measurement setup was also utilized, where spectra were taken with one well-shielded unsuppressed Ge detector in a low-background environment. In addition, for reference purposes an in-beam measurement of $3 \mathrm{~h}$ was also performed using a $535-\mathrm{MeV}{ }^{134} \mathrm{Xe}$ beam, with the ${ }^{48} \mathrm{Ca}$-oxide target in the center of the $8 \pi$ detector array.

\section{DATA ANALYSIS}

There are only few examples of $K$ isomers decaying through $\beta$ decay in the $A \approx 180$ region [17], and all of them have spin less than 12. In the quantitative analysis of the nuclei studied in the present work we assume that any new isomer populated would decay through $\gamma$-ray transitions. Even if the first step of a high- $K$ isomer's decay were by $\beta$ emission, this would be followed by $\gamma$-ray emission. We have found no evidence for new $\beta$-decaying isomers and in this work it is the $\gamma$-ray emission that we quantify.

The number of counts from a $\gamma$-ray transition deexciting (directly or indirectly) an isomeric level populated in a beam irradiation is described by

$$
A_{\gamma}=\tau\left(1-e^{-T_{\text {irrad }} / \tau}\right)\left(e^{-t_{1} / \tau}-e^{-t_{2} / \tau}\right) \frac{\sigma N_{t} I \epsilon f_{\mathrm{ex}} B_{R}}{(1+\alpha)}
$$

where $\tau$ is the mean life of the isomer; $T_{\text {irrad }}$ is the irradiation time; $t_{1}$ is the time between the end of the irradiation and start of the measurement; $t_{2}$ is the time between the end of the irradiation and end of the measurement; $\alpha$ is the electron conversion coefficient; $B_{R}$ is the branching ratio of the transition; $\epsilon$ is the $\gamma$-ray efficiency; $I$ is the beam intensity; $\sigma$ is the total production cross section of the nucleus; $N_{t}$ is the number of target nuclei per unit area; and $f_{\text {ex }}$ is the cross section of the isomer compared with the total cross section of the nucleus, multiplied with the percentage of the isomer deexcitation passing through the level decaying by the transition under analysis. In the case where a $\gamma$-ray transition directly deexcites an isomer, $f_{\text {ex }}$ is simply the fraction of the production cross section of the isomeric state compared to the total. (All the quantities listed in the equation have to be given in International System units.)
Since there are large uncertainties in $N_{t}, \sigma, I$, and absolute $\epsilon$, it is better to compare $\gamma$-ray line intensities to reference transitions deexciting $\beta$-unstable nuclei or known isomers for which production is specific to the target (and not to the beam). The function $F(\tau)$ is defined as

$$
F(\tau)=\tau\left(1-e^{-T_{\text {irrad }} / \tau}\right)\left(e^{-t_{1} / \tau}-e^{-t_{2} / \tau}\right)
$$

and it gives the sensitivity of the measurement as a function of the mean life of the isomeric decay. In the case when the $\gamma$-ray transition which deexcites an isomer is not observed, then one can say $A_{\gamma}<N \sqrt{2 \cdot \text { background, where }}$ $\sqrt{2 \cdot \text { background }}$ is the standard deviation of the area for an unobserved peak and $N$ is the number of standard deviations. Limits for the lifetime can be deduced using the formula derived from Eqs. (1) and (2)

$$
F(\tau)<\frac{N \sqrt{2 \cdot \text { background }}(1+\alpha)}{\epsilon f_{\mathrm{ex}} B_{R}}\left(\frac{\epsilon f_{\mathrm{ex}} B_{R}}{A_{\gamma}(1+\alpha)}\right) \frac{\sigma_{\text {ref }}}{\sigma} F(\tau)_{\text {ref }} .
$$

The strongest $\beta$ decays observed were from nuclei populated in the particle-transfer channels. Consequently, the $\gamma$-ray spectra are dominated, in addition to the W-Ta-Hf decay transitions, by the transitions following the $\beta$ decay of the nuclei close in $N$ and $Z$ to ${ }^{134,136} \mathrm{Xe}$ and ${ }^{48} \mathrm{Ca}$. The 489 and $808 \mathrm{keV} \gamma$-ray transitions that follow the $\beta$ decay of ${ }^{47} \mathrm{Ca}, T_{1 / 2}=4.536 \mathrm{~d}$ [17] were chosen as reference lines. The intensities of these transitions are related to the quantity of ${ }^{48} \mathrm{Ca}$ in the target. The ${ }^{47} \mathrm{Ca}$ is produced from ${ }^{48} \mathrm{Ca}$ in one-neutron knockout reactions and from the decay of ${ }^{47} \mathrm{~K}$, this latter produced from ${ }^{48} \mathrm{Ca}$ by knocking out one proton. Since the half life of ${ }^{47} \mathrm{~K}, T_{1 / 2}=17 \mathrm{~s}$, is very short compared with the time between the end of an irradiation and the start of a measurement, it can be considered that the chosen reference $\gamma$ rays have the $4.5 \mathrm{~d}$ lifetime of ${ }^{47} \mathrm{Ca}$. The consistency of the results obtained was checked by using additional reference $\gamma$-ray transitions, following the $\beta$ decay of ${ }^{48} \mathrm{Sc}\left(T_{1 / 2}=43.67 \mathrm{~h}\right),{ }^{177} \mathrm{~W}\left(T_{1 / 2}=2.25 \mathrm{~h}\right) \quad[17]$, and ${ }^{177} \mathrm{Hf}\left(T_{1 / 2}=51 \mathrm{~min}, K=37 / 2\right.$ isomer) [18]. It should be noted that decays with $\gamma$-ray multiplicity of one cannot be used from the data collected with the $8 \pi$ spectrometer, due to the coincidence requirement imposed.

\section{RESULTS}

The main characteristics of the measurements performed are listed in Table I. It should be noted that target 3 received the highest beam intensity for the longest period. Compared with target 2, however, we found that the reference lines are weaker, probably because target 3 was partially damaged by the higher beam. Spectrum numbers 1 and 5 are shown in Fig. 1.

In order to place limits on the lifetimes of the possible isomers, it is necessary to know the relative production cross sections of the nuclei [see Eq. (3)]. These have been calculated using two different programs: PACE [19] and CASCADE [20]. The differences in the results obtained with the two codes can be large, and as previous experience also shows, 
TABLE I. Characteristics of the off-beam measurements.

\begin{tabular}{lcccccccc}
\hline \hline Spectrum No. & Beam & $E_{\text {beam }}(\mathrm{MeV})$ & $I_{\text {beam }}(\mathrm{pnA})$ & Target No. & $T_{\text {irrad }}(\mathrm{h})$ & $t_{1}(\mathrm{~h})$ & $t_{2}(\mathrm{~h})$ & Detector \\
\hline 1 & ${ }^{134} \mathrm{Xe}$ & 535 & 6 & 4 & 20 & 0.5 & 20.5 & $8 \pi$ \\
2 & ${ }^{134} \mathrm{Xe}$ & 535 & 6 & 5 & 20 & 7 & 24 & $8 \pi$ \\
3 & ${ }^{134} \mathrm{Xe}$ & 535 & 6 & 4 & 20 & 22.5 & 27 & $8 \pi$ \\
4 & ${ }^{134} \mathrm{Xe}$ & 535 & 6 & 5 & 20 & 49.5 & 74.3 & $1 \mathrm{Ge}$ \\
5 & ${ }^{136} \mathrm{Xe}$ & 550 & 6 & 1 & 2 & 0.5 & 12.5 & $8 \pi$ \\
6 & ${ }^{136} \mathrm{Xe}$ & 550 & 8 & 2 & 10 & 1.7 & 3.2 & $8 \pi$ \\
7 & ${ }^{136} \mathrm{Xe}$ & 550 & 8 & 2 & 10 & 3.2 & 22.2 & $8 \pi$ \\
8 & ${ }^{136} \mathrm{Xe}$ & 550 & 8 & 2 & 10 & 22.2 & 25.2 & $1 \mathrm{Ge}$ \\
9 & ${ }^{136} \mathrm{Xe}$ & 535 & 15 & 3 & 22 & 54 & 70.8 & $1 \mathrm{Ge}$ \\
\hline \hline
\end{tabular}

sometimes far from the measured values. Due to this, cross sections determined from the present experiment were used where possible, and where it was not possible, the assumptions used will be discussed for the individual nuclei.

By determining the intensity of the $\gamma$ rays and using Eq. (1), relative cross sections can be determined for some cases. From the in-beam measurement of the ${ }^{48} \mathrm{Ca}+{ }^{134} \mathrm{Xe}$ reaction at $535 \mathrm{MeV}$, the relative production cross sections obtained were $\sigma\left({ }^{177} \mathrm{~W}\right)_{\text {rel }}=45(13), \sigma\left({ }^{178} \mathrm{~W}\right)_{\text {rel }}=100$, and $\sigma\left({ }^{179} \mathrm{~W}\right)_{\text {rel }}$ $=39(5)$ for the tungsten isotopes. Analysis of the $\beta$ and $K$-isomer decay spectra gave cross-section ratios of $\sigma\left({ }^{177} \mathrm{~W}\right) / \sigma\left({ }^{178} \mathrm{Ta}\right)=85(15), \quad \sigma\left({ }^{177} \mathrm{~W}\right) / \sigma\left({ }^{48} \mathrm{Sc}\right)=75(11)$, $\sigma\left({ }^{177} \mathrm{~W}\right) / \sigma\left({ }^{47} \mathrm{Ca}+{ }^{47} \mathrm{~K}\right)=1.35(15)$ from the ${ }^{134} \mathrm{Xe}$ irradiated targets; and $\sigma\left({ }^{47} \mathrm{Ca}+{ }^{47} \mathrm{~K}\right) / \sigma\left({ }^{177} \mathrm{Hf}, K=37 / 2\right)=130(16)$, $\sigma\left({ }^{47} \mathrm{Ca}+{ }^{47} \mathrm{~K}\right) / \sigma\left({ }^{48} \mathrm{Sc}\right)=62(7)$ from the ${ }^{136} \mathrm{Xe}$ irradiated targets. The yield of ${ }^{178} \mathrm{Ta}$ refers to the population of the $7^{-}$ ground state (i.e., the population of the $1^{+}$isomer, close to the ground state but at unknown energy, is not included) and ${ }^{177} \mathrm{Hf}$ refers to the population of the $K=37 / 2, T_{1 / 2}=51 \mathrm{~m}$ state [18]. In all the other cases the ground-state population is the reference. The $\sigma\left({ }^{47} \mathrm{Ca}+{ }^{47} \mathrm{~K}\right) / \sigma\left({ }^{48} \mathrm{Sc}\right)$ value is the same for both the ${ }^{134} \mathrm{Xe}$ and ${ }^{136} \mathrm{Xe}$ beams and for the two beam energies $\left({ }^{136} \mathrm{Xe}\right)$, at least within the quoted uncertainties. The relative cross sections of other nuclei could not be determined from the off-beam experiment for different reasons: either the nucleus is stable, the lifetime is too short, the $\gamma$-ray multiplicity is unity, or the nucleus is also produced in the decay of other nuclei. The relative cross-section values used are listed in Table II. These values will be discussed in conjunction with the individual nuclei.

The observation of the known $51 \mathrm{~m}, K=37 / 2$ isomer in ${ }^{177} \mathrm{Hf}[18]$ is of particular note. The $51 \mathrm{~m}$ half life is at the lower end of the range of sensitivity of the present search. With its high- $K$ value of $37 / 2$, and the requirement of an $\alpha$-particle evaporation for its formation, this isomer serves as a sensitive test for the validity of the present measurements. In Fig. 1 (lower part) the $\gamma$-ray transitions at 278, 295, 311, and $326 \mathrm{keV}$ are clear evidence for its formation. It is also notable that the ${ }^{178} \mathrm{Ta} 7^{-}$ground state can only be populated through the proton-evaporation channel (and not from the $\beta$ decay of ${ }^{178} \mathrm{~W}$ ) so that the intensity of its decay (by the 213 and $326 \mathrm{keV}$ transitions seen in the upper part of Fig. 1)

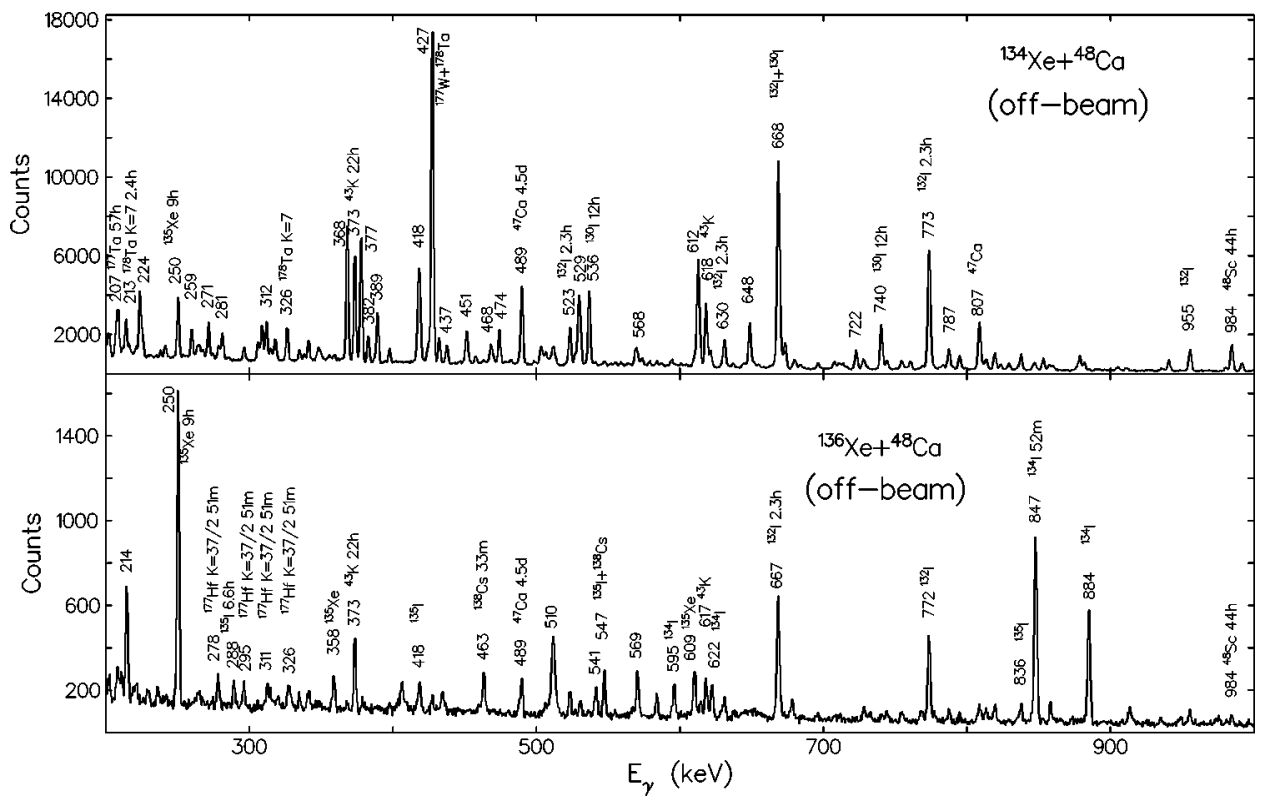

FIG. 1. Spectrum numbers 1 and 5 (see Table I). In the upper spectrum the peaks labeled only by energy are $\gamma$-ray transitions following the $\beta$ decay of ${ }^{177} \mathrm{~W}, T_{1 / 2}=2.25 \mathrm{~h}$. 
TABLE II. The relative cross-section values used in the ${ }^{134} \mathrm{Xe}$ and ${ }^{136} \mathrm{Xe}$ induced reactions.

\begin{tabular}{|c|c|c|c|c|c|c|c|c|}
\hline \multirow[t]{2}{*}{$Z$} & \multicolumn{4}{|c|}{$\begin{array}{c}{ }^{134} \text { Xe beam } \\
N\end{array}$} & \multicolumn{4}{|c|}{$\begin{array}{c}{ }^{136} \text { Xe beam } \\
N\end{array}$} \\
\hline & 103 & 104 & 105 & 106 & 105 & 106 & 107 & 108 \\
\hline 74 & 45 & 100 & 39 & & & 100 & 39 & \\
\hline 73 & & 0.24 & 0.53 & 0.21 & & & 0.53 & 0.21 \\
\hline 72 & 1.06 & 0.42 & & & 1.06 & 0.42 & & \\
\hline
\end{tabular}

gives a reliable measure of the relative proton-channel cross section.

The $F(\tau)$ values, as a function of the mean life, are plotted in Fig. 2 for the first four measurements $\left({ }^{134} \mathrm{Xe}\right.$ beam) and in Fig. 3 for spectrum numbers $5-9$ ( ${ }^{136} \mathrm{Xe}$ beam).

Transitions from the lower parts of the known level schemes were searched for in the spectra, since any purported, high-lying isomer should decay through these levels. This is comparatively simple in the case of the even-even nuclei, where there are not many paths available, but is much more complicated in the case of the odd and odd-odd nuclei. Low-lying high- $K$ isomers are known in many nuclei of this region, and it is very probable that any new isomers with lifetimes in the region of hours would decay through the known isomers. For this reason the presence of $\gamma$-ray transitions from the decay of known isomers was also checked.

Table III gives the limits of the deduced $F(\tau)$ values and the corresponding mean-life limits for transitions which were considered to be significant. The lack of a limit for certain spectra is due to contaminant lines. The low-energy transitions have the difficulty of low intensity because of high electron conversion coefficients, and low detector efficiency in the measurements with the $8 \pi$ spectrometer due to the use of absorbers. The $F(\tau)$ and $\tau$ limits were obtained taking $f_{\text {ex }}=0.01$ for the tungsten isotopes, and $f_{\text {ex }}=0.1$ for the tantalum and hafnium nuclei populated after the evaporation of

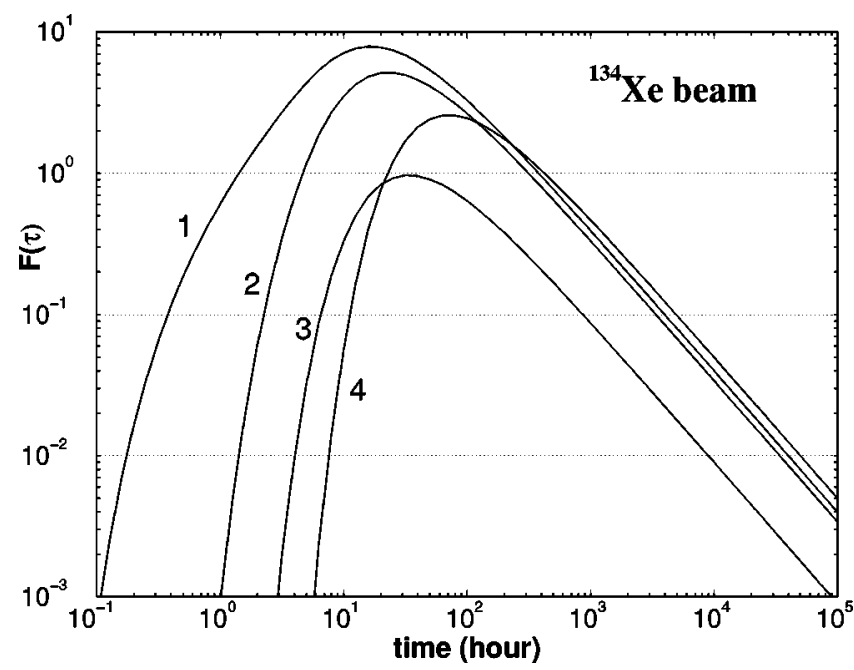

FIG. 2. $F(\tau)$ as function of the mean life for spectrum numbers $1-4$. For the definition of $F(\tau)$ and the spectra properties, see text and Table I, respectively.

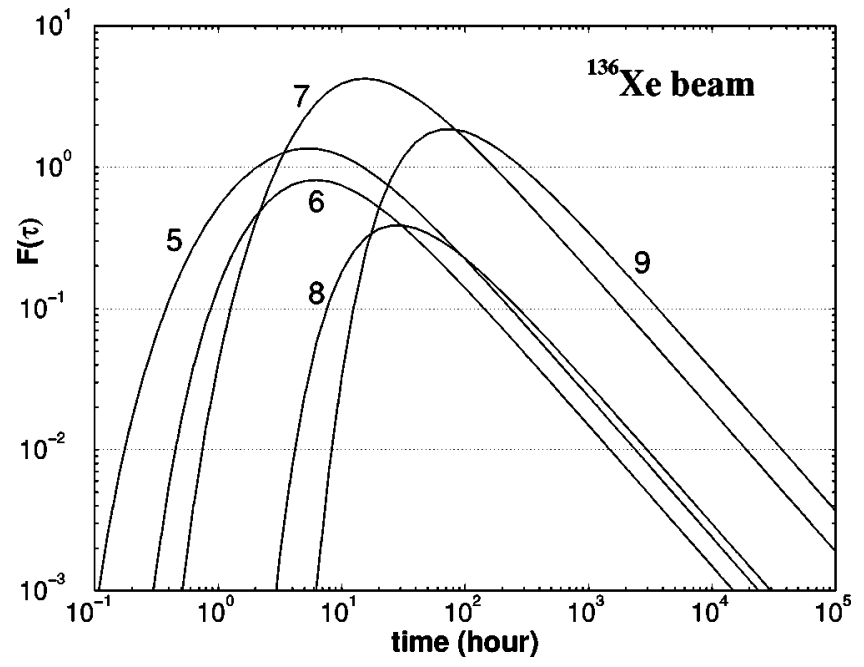

FIG. 3. $F(\tau)$ as a function of the mean life for spectrum numbers 5-9. For the definition of $F(\tau)$ and the spectra properties, see text and Table 1 , respectively.

charged particles. In order to obtain reliable limits, we used $N=5$ for the number of standard deviations. This high value corresponds to the probability of $>99 \%$ that there is no isomer with a lifetime within the excluded region, even in the case of an unlikely error of $50 \%$ in the cross-section assumption.

If one were to take an isomer population different from $1 \%\left(f_{\text {ex }}=0.01\right)$ relative to a tungsten ground state (or $10 \%$ for tantalum and hafnium) the new $F(\tau)$ limit would be calculated as $F(\tau)=(F(\tau))_{\text {tab.3 }} /\left(100 * f_{\text {ex }}\right)$ for tungsten, and $F(\tau)=(F(\tau))_{\text {tab. } 3} /\left(10 * f_{\text {ex }}\right)$ for tantalum and hafnium. The corresponding mean-life limits can be obtained using Figs. 2 and 3.

In the following sections we discuss the nuclei studied individually, starting with those populated in the ${ }^{134} \mathrm{Xe}$ induced reaction and following with the nuclei populated in the ${ }^{136} \mathrm{Xe}$ induced reaction. In some cases, the lower lifetime limit is already excluded by in-beam measurements (independent of the present work) and it is the upper limits on lifetimes that are of principal interest.

\section{A. ${ }^{178} \mathbf{W}$}

The nucleus ${ }^{178} \mathrm{~W}$ is the main product in the ${ }^{134} \mathrm{Xe}$ $+{ }^{48} \mathrm{Ca}$ reaction. The relative cross section has been determined from the experiment. From Table III one can establish that if an isomer had been populated with an intensity of $1 \%$ of the total population of the nucleus, and it decayed through the $106 \mathrm{keV}, 2^{+} \rightarrow 0^{+}$transition, its mean life would have to be less than $1.3 \mathrm{~h}$ or more than $1200 \mathrm{~h}(50 \mathrm{~d})$. If such an isomer had decayed into the yrast band at the $6^{+}$state or higher, its mean life cannot be between $0.5 \mathrm{~h}$ and $350 \mathrm{~d}$. If it decays into other bands which depopulate through the $2^{-}$ $\rightarrow 2^{+} 939-\mathrm{keV}$ transition, the isomer mean life cannot be between $6 \mathrm{~h}$ and $200 \mathrm{~d}$. Assuming a population of only $0.1 \%$, the mean life of an isomer which decays to the higher part of the yrast band could not be between $2 \mathrm{~h}$ and $30 \mathrm{~d}$. In contrast, considering such an isomer with a population of $10 \%$, the mean life cannot be between 15 min and $97 \mathrm{yr}$. 
TABLE III. The deduced mean-life limits considering $N=5$ and $f_{\mathrm{ex}}=0.01$ for tungsten isotopes and $f_{\text {ex }}=0.1$ for tantalum and hafnium isotopes. The first and second parts of the table refer to the nuclei produced with the ${ }^{134} \mathrm{Xe}$ and ${ }^{136} \mathrm{Xe}$ beams, respectively. For further explanations see the text.

\begin{tabular}{|c|c|c|c|c|}
\hline Nucleus/Reference(s) & $E_{\gamma}(\mathrm{keV})$ & Spectrum No. ${ }^{a}$ & $F(\tau)$ & 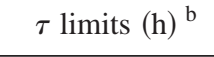 \\
\hline \multirow[t]{6}{*}{${ }^{178} \mathrm{~W}[6]$} & $106\left(2^{+} \rightarrow 0^{+}\right)$ & 1 & $9.0 \times 10^{-1}$ & $<1.3$ or $>430$ \\
\hline & & 4 & $4.0 \times 10^{-1}$ & $<16$ or $>1200$ \\
\hline & $237\left(4^{+} \rightarrow 2^{+}\right)$ & 4 & $5.8 \times 10^{-2}$ & $<10$ or $>8500$ \\
\hline & $351\left(6^{+} \rightarrow 4^{+}\right)$ & 1 & $1.7 \times 10^{-1}$ & $<0.5$ or $>2300$ \\
\hline & $939\left(2^{-} \rightarrow 2^{+}\right)$ & 3 & $7.9 \times 10^{-2}$ & $<6.2$ or $>1100$ \\
\hline & & 4 & $1.0 \times 10^{-1}$ & $<11$ or $>4800$ \\
\hline \multirow[t]{6}{*}{${ }^{177} \mathrm{~W}[22]$} & $197\left(7 / 2^{-} \rightarrow 3 / 2^{-}\right)$ & 1 & $4.0 \times 10^{-1}$ & $<0.8$ or $>990$ \\
\hline & $85\left(7 / 2^{+} \rightarrow 5 / 2^{-}\right)$ & 1 & $2.3 \times 10^{0}$ & $<2.8$ or $>150$ \\
\hline & & 4 & $2.4 \times 10^{-1}$ & $<14$ or $>2000$ \\
\hline & $131\left(9 / 2^{-} \rightarrow 7 / 2^{-}\right)$ & 2 & $1.0 \times 10^{0}$ & $<4.7$ or $>310$ \\
\hline & $256\left(11 / 2^{-} \rightarrow 7 / 2^{-}\right)$ & 1 & $5.3 \times 10^{-1}$ & $<0.9$ or $>730$ \\
\hline & & 4 & $1.8 \times 10^{-1}$ & $<13$ or $>2700$ \\
\hline \multirow[t]{14}{*}{${ }^{179} \mathrm{~W}[23]$} & $189\left(9 / 2^{+} \rightarrow 9 / 2^{-}\right)$ & 1 & $1.4 \times 10^{0}$ & $<1.9$ or $>260$ \\
\hline & & 4 & $1.2 \times 10^{-1}$ & $<12$ or $>4000$ \\
\hline & $253\left(11 / 2^{+} \rightarrow 9 / 2^{-}\right)$ & 1 & $8.9 \times 10^{-1}$ & $<1.3$ or $>430$ \\
\hline & & 2 & $7.0 \times 10^{-1}$ & $<4.0$ or $>460$ \\
\hline & $204\left(7 / 2^{-} \rightarrow 3 / 2^{-}\right)$ & 1 & $1.0 \times 10^{0}$ & $<1.5$ or $>370$ \\
\hline & & 4 & $1.0 \times 10^{-1}$ & $<11$ or $>4800$ \\
\hline & $215\left(9 / 2^{-} \rightarrow 5 / 2^{-}\right)$ & 3 & $9.5 \times 10^{-2}$ & $<6.5$ or $>920$ \\
\hline & & 4 & $1.7 \times 10^{-1}$ & $<13$ or $>2900$ \\
\hline & $402\left(17 / 2^{-} \rightarrow 13 / 2^{-}\right)$ & 1 & $2.8 \times 10^{-1}$ & $<0.6$ or $>1400$ \\
\hline & $442\left(19 / 2^{-} \rightarrow 15 / 2^{-}\right)$ & 1 & $2.8 \times 10^{-1}$ & $<0.6$ or $>1400$ \\
\hline & $313\left(13 / 2^{-} \rightarrow 9 / 2^{-}\right)$ & 3 & $9.4 \times 10^{-2}$ & $<6.4$ or $>930$ \\
\hline & & 4 & $1.6 \times 10^{-1}$ & $<12$ or $>3000$ \\
\hline & $359\left(15 / 2^{-} \rightarrow 11 / 2^{-}\right)$ & 3 & $9.3 \times 10^{-2}$ & $<6.4$ or $>930$ \\
\hline & & 4 & $1.3 \times 10^{-1}$ & $<12$ or $>3700$ \\
\hline \multirow[t]{5}{*}{${ }^{177} \mathrm{Ta}[8]$} & $312\left(21 / 2^{-} \rightarrow 19 / 2^{-}\right)$ & 3 & $1.7 \times 10^{0}$ & - \\
\hline & & 4 & $3.2 \times 10^{0}$ & - \\
\hline & $218\left(17 / 2^{-} \rightarrow 15 / 2^{-}\right)$ & 1 & $1.5 \times 10^{1}$ & - \\
\hline & & 2 & $8.8 \times 10^{0}$ & - \\
\hline & & 4 & $6.0 \times 10^{0}$ & - \\
\hline \multirow[t]{9}{*}{${ }^{178} \mathrm{Ta}[24]$} & $198\left(8^{-} \rightarrow 7^{-}\right)$ & 2 & $1.4 \times 10^{0}$ & $<5.4$ or $>220$ \\
\hline & $219\left(9^{-} \rightarrow 8^{-}\right)$ & 4 & $1.5 \times 10^{0}$ & $<29$ or $>250$ \\
\hline & $289\left(6^{-} \rightarrow 7^{-}\right)$ & 1 & $2.7 \times 10^{0}$ & $<3.2$ or $>120$ \\
\hline & & 2 & $1.4 \times 10^{0}$ & $<5.4$ or $>220$ \\
\hline & $194\left(9^{-} \rightarrow 8^{-}\right)$ & 2 & $3.3 \times 10^{0}$ & $<9.5$ or $>73$ \\
\hline & $392\left(9^{-} \rightarrow 7^{-}\right)$ & 1 & $6.2 \times 10^{0}$ & $<7.7$ or $>39$ \\
\hline & & 2 & $4.0 \times 10^{0}$ & $<12$ or $>52$ \\
\hline & $220\left(8^{+} \rightarrow 7^{-}\right)$ & 3 & $5.4 \times 10^{-1}$ & $<13$ or $>130$ \\
\hline & & 4 & $7.3 \times 10^{-1}$ & $<20$ or $>600$ \\
\hline \multirow[t]{3}{*}{${ }^{179} \mathrm{Ta}[21]$} & $232\left(21 / 2^{-} \rightarrow 19 / 2^{-}\right)$ & 1 & $1.5 \times 10^{1}$ & - \\
\hline & & 2 & $8.3 \times 10^{0}$ & - \\
\hline & $475\left(21 / 2^{-} \rightarrow 17 / 2^{-}\right)$ & 3 & $8.9 \times 10^{0}$ & - \\
\hline \multirow[t]{6}{*}{${ }^{175} \mathrm{Hf}[25]$} & $104\left(9 / 2^{-} \rightarrow 7 / 2^{-}\right)$ & 3 & $9.0 \times 10^{-1}$ & $<24$ or $>51$ \\
\hline & $127\left(11 / 2^{-} \rightarrow 9 / 2^{-}\right)$ & 4 & $2.1 \times 10^{0}$ & $<39$ or $>150$ \\
\hline & $231\left(11 / 2^{-} \rightarrow 7 / 2^{-}\right)$ & 1 & $1.3 \times 10^{1}$ & - \\
\hline & & 2 & $6.8 \times 10^{0}$ & - \\
\hline & & 4 & $3.8 \times 10^{0}$ & - \\
\hline & $126\left(7 / 2^{+} \rightarrow 7 / 2^{-}\right)$ & 4 & $5.4 \times 10^{0}$ & - \\
\hline
\end{tabular}


TABLE III. (Continued).

\begin{tabular}{|c|c|c|c|c|}
\hline Nucleus/Reference(s) & $E_{\gamma}(\mathrm{keV})$ & Spectrum No. ${ }^{a}$ & $F(\tau)$ & $\tau$ limits (h) ${ }^{b}$ \\
\hline \multirow[t]{5}{*}{${ }^{176} \mathrm{Hf}[26]$} & $307\left(6^{+} \rightarrow 4^{+}\right)$ & 3 & $7.8 \times 10^{-1}$ & $<18$ or $>71$ \\
\hline & & 4 & $1.2 \times 10^{0}$ & $<25$ or $>330$ \\
\hline & $401\left(8^{+} \rightarrow 6^{+}\right)$ & 1 & $3.4 \times 10^{0}$ & $<4.0$ or $>94$ \\
\hline & & 2 & $2.3 \times 10^{0}$ & $<7.2$ or $>120$ \\
\hline & $1043\left(6^{+} \rightarrow 4^{+}\right)$ & 1 & $7.6 \times 10^{0}$ & $<12$ or $>22$ \\
\hline \multirow[t]{6}{*}{${ }^{180} \mathrm{~W}[27]$} & $104\left(2^{+} \rightarrow 0^{+}\right)$ & 5 & $5.4 \times 10^{-1}$ & $<1.0$ or $>37$ \\
\hline & & 7 & $1.3 \times 10^{0}$ & $<3.5$ or $>130$ \\
\hline & $234\left(4^{+} \rightarrow 2^{+}\right)$ & 6 & $4.4 \times 10^{-2}$ & $<0.7$ or $>330$ \\
\hline & & 7 & $7.5 \times 10^{-2}$ & $<1.2$ or $>2500$ \\
\hline & $351\left(6^{+} \rightarrow 4^{+}\right)$ & 5 & $1.2 \times 10^{-1}$ & $<0.4$ or $>190$ \\
\hline & & 7 & $1.3 \times 10^{-1}$ & $<1.4$ or $>1400$ \\
\hline \multirow[t]{13}{*}{${ }^{181} \mathrm{~W}[28]$} & $113\left(11 / 2^{+} \rightarrow 9 / 2^{+}\right)$ & 6 & $6.6 \times 10^{-1}$ & $<3.1$ or $>13$ \\
\hline & & 7 & $2.8 \times 10^{0}$ & $<6.1$ or $>48$ \\
\hline & $137\left(13 / 2^{+} \rightarrow 11 / 2^{+}\right)$ & 6 & $3.3 \times 10^{-1}$ & $<1.6$ or $>37$ \\
\hline & & 9 & $6.7 \times 10^{-1}$ & $<23$ or $>470$ \\
\hline & $301\left(15 / 2^{+} \rightarrow 11 / 2^{+}\right)$ & 5 & $4.4 \times 10^{-1}$ & $<0.9$ or $>46$ \\
\hline & & 7 & $4.5 \times 10^{-1}$ & $<2.1$ or $>400$ \\
\hline & $348\left(17 / 2^{+} \rightarrow 13 / 2^{+}\right)$ & 5 & $4.3 \times 10^{-1}$ & $<0.9$ or $>48$ \\
\hline & & 9 & $5.4 \times 10^{-1}$ & $<20$ or $>610$ \\
\hline & $119\left(9 / 2^{-} \rightarrow 7 / 2^{-}\right)$ & 6 & $5.6 \times 10^{-1}$ & $<2.5$ or $>18$ \\
\hline & $266\left(11 / 2^{-} \rightarrow 7 / 2^{-}\right)$ & 5 & $5.5 \times 10^{-1}$ & $<1.0$ or $>35$ \\
\hline & & 9 & $1.2 \times 10^{0}$ & $<32$ or $>220$ \\
\hline & $320\left(13 / 2^{-} \rightarrow 9 / 2^{-}\right)$ & 6 & $1.2 \times 10^{-1}$ & $<0.9$ or $>120$ \\
\hline & & 7 & $3.6 \times 10^{-1}$ & $<1.9$ or $>510$ \\
\hline \multirow[t]{6}{*}{${ }^{180} \mathrm{Ta}[29,30]$} & $203\left(10^{-} \rightarrow 9^{-}\right)$ & 5 & $4.8 \times 10^{0}$ & - \\
\hline & & 9 & $3.2 \times 10^{0}$ & - \\
\hline & $225\left(11^{-} \rightarrow 10^{-}\right)$ & 6 & $7.5 \times 10^{-1}$ & $<4.1$ or $>9.5$ \\
\hline & $269\left(13^{-} \rightarrow 12^{-}\right)$ & 7 & $4.0 \times 10^{0}$ & $<11$ or $>23$ \\
\hline & $516\left(13^{-} \rightarrow 11^{-}\right)$ & 7 & $3.5 \times 10^{0}$ & $<7.9$ or $>33$ \\
\hline & $432\left(15^{-} \rightarrow 13^{-}\right)$ & 5 & $4.7 \times 10^{0}$ & - \\
\hline \multirow[t]{11}{*}{${ }^{181} \mathrm{Ta}[28,4]$} & $136\left(9 / 2^{+} \rightarrow 7 / 2^{+}\right)$ & 5 & $2.9 \times 10^{1}$ & - \\
\hline & & 7 & $2.2 \times 10^{1}$ & - \\
\hline & $302\left(11 / 2^{+} \rightarrow 7 / 2^{+}\right)$ & 6 & $6.7 \times 10^{0}$ & - \\
\hline & & 7 & $2.0 \times 10^{1}$ & - \\
\hline & $415\left(15 / 2^{+} \rightarrow 11 / 2^{+}\right)$ & 5 & $9.4 \times 10^{0}$ & - \\
\hline & & 9 & $8.59 \times 10^{0}$ & - \\
\hline & $152\left(11 / 2^{-} \rightarrow 9 / 2^{-}\right)$ & 5 & $1.9 \times 10^{1}$ & - \\
\hline & $179\left(13 / 2^{-} \rightarrow 11 / 2^{-}\right)$ & 5 & $7.4 \times 10^{0}$ & - \\
\hline & & 7 & $7.9 \times 10^{0}$ & - \\
\hline & $205\left(17 / 2^{-} \rightarrow 15 / 2^{-}\right)$ & 6 & $2.6 \times 10^{0}$ & - \\
\hline & & 9 & $6.3 \times 10^{0}$ & - \\
\hline \multirow[t]{7}{*}{${ }^{177} \mathrm{Hf}[13]$} & $366\left(39 / 2^{+} \rightarrow 37 / 2^{-}\right)$ & 5 & $6.4 \times 10^{-1}$ & $<1.2$ or $>29$ \\
\hline & & 7 & $6.5 \times 10^{-1}$ & $<2.4$ or $>270$ \\
\hline & $401\left(39 / 2^{-} \rightarrow 37 / 2^{-}\right)$ & 6 & $3.4 \times 10^{-1}$ & $<1.6$ or $>36$ \\
\hline & & 7 & $8.1 \times 10^{-1}$ & $<2.7$ or $>220$ \\
\hline & $360\left(41 / 2^{+} \rightarrow 39 / 2^{+}\right)$ & 9 & $1.2 \times 10^{0}$ & $<31$ or $>230$ \\
\hline & $421\left(41 / 2^{-} \rightarrow 39 / 2^{-}\right)$ & 5 & $9.3 \times 10^{-1}$ & $<1.8$ or $>17$ \\
\hline & & 7 & $9.5 \times 10^{-1}$ & $<2.9$ or $>180$ \\
\hline \multirow[t]{2}{*}{${ }^{178} \mathrm{Hf}[31,32]$} & $93\left(2^{+} \rightarrow 0^{+}\right)$ & 6 & $1.2 \times 10^{1}$ & - \\
\hline & & 8 & $5.9 \times 10^{0}$ & - \\
\hline
\end{tabular}


TABLE III. (Continued).

\begin{tabular}{ccccc}
\hline \hline Nucleus/Reference(s) & $E_{\gamma}(\mathrm{keV})$ & Spectrum No. ${ }^{\mathrm{a}}$ & $F(\tau)$ & $\tau$ limits $(\mathrm{h})^{\mathrm{b}}$ \\
\hline $213\left(4^{+} \rightarrow 2^{+}\right)$ & 9 & $2.1 \times 10^{0}$ & - \\
$326\left(6^{+} \rightarrow 4^{+}\right)$ & 8 & $4.6 \times 10^{-1}$ & - \\
$426\left(8^{+} \rightarrow 6^{+}\right)$ & 8 & $5.7 \times 10^{-1}$ & - \\
$580\left(12^{+} \rightarrow 10^{+}\right)$ & 6 & $6.7 \times 10^{-1}$ & $<3.2$ or $>13$ \\
& 7 & $1.9 \times 10^{0}$ & $<4.4$ or $>81$ \\
& $721\left(6^{+} \rightarrow 6^{+}\right)$ & 7 & $3.7 \times 10^{0}$ & $<9.0$ or $>28$ \\
$357\left(17^{+} \rightarrow 16^{+}\right)$ & 9 & $3.1 \times 10^{0}$ & - \\
$377\left(18^{+} \rightarrow 17^{+}\right)$ & 7 & $2.1 \times 10^{0}$ & $<4.8$ or $>69$ \\
\hline
\end{tabular}

${ }^{\mathrm{a}}$ For the characterization of the spectra see Table II.

${ }^{\mathrm{b}}$ The lifetime of the isomer cannot be between the given two values under the applied considerations.

$$
\text { B. }{ }^{177} \mathbf{W}
$$

The level schemes of the odd nuclei are more complex than those of the even-even nuclei, therefore the presence of several transitions in the spectra was sought in these cases. Since the discussion for every $\gamma$-ray transition would be lengthy, and the information is contained in Table III and Figs. 2 and 3, only a brief comment on the lifetime limits is made here. For ${ }^{177} \mathrm{~W}$ no isomer with a mean life between $3 \mathrm{~h}$ and $41 \mathrm{~d}$ can be present under our assumptions. The relative cross sections are from the experiment.

$$
\text { C. }{ }^{179} \mathrm{~W}
$$

The relative cross sections are from the experiment. No isomer with mean life between $6 \mathrm{~h}$ and $19 \mathrm{~d}$ can be in ${ }^{179} \mathrm{~W}$. As can be seen from Table III, the half-life limits are in the same range for ${ }^{177} \mathrm{~W},{ }^{178} \mathrm{~W}$, and ${ }^{179} \mathrm{~W}$, since the cross sections are similar.

According to the calculations, ${ }^{179} \mathrm{~W}$ was also populated in the ${ }^{136} \mathrm{Xe}$ induced reaction with a similar cross section. However, since there is not an experimentally determined cross section for this case and the calculated values have large uncertainties, half-life limits have not been evaluated.

$$
\text { D. }{ }^{177} \mathbf{T a}
$$

The tantalum nuclei were populated after the evaporation of one proton (and some neutrons), so their cross sections are much lower than those leading to the tungsten nuclei. For ${ }^{177}$ Ta experimental cross sections could not be obtained, so it was assumed that $\sigma\left({ }^{177} \mathrm{~W}\right) / \sigma\left({ }^{177} \mathrm{Ta}\right)=\sigma\left({ }^{178} \mathrm{~W}\right) / \sigma\left({ }^{178} \mathrm{Ta}\right)$. This assumption seems to be reasonable, but since the extracted lifetime limits are very sensitive to the cross sections, the results obtained have to be treated with caution.

In the ${ }^{177} \mathrm{Ta}$ nucleus, a low-lying $K=21 / 2$ isomer is known [8] with a level energy of $1355 \mathrm{keV}$. In Table III only the $\gamma$-ray transitions depopulating this isomer are listed. However, lifetime limits for higher lying isomers cannot be given with the present assumptions. Considering a higher $f_{\mathrm{ex}}$ value, i.e., stronger isomer population, useful results can nevertheless be obtained based on the listed $F(\tau)$ limits. For example, the mean life of an isomer in ${ }^{177}$ Ta populated at the level of $f_{\text {ex }}=0.3$, cannot be between $11 \mathrm{~h}$ and $16 \mathrm{~d}$, otherwise it would have been detected.

\section{E. ${ }^{178} \mathrm{Ta}$}

The production cross section was determined from the experiment: $\sigma\left({ }^{178} \mathrm{Ta}\right)=\sigma\left({ }^{178} \mathrm{~W}\right) / 183(32)$, without taking into account the population of the $1^{+}$isomer in ${ }^{178} \mathrm{Ta}$. Due to the fact that ${ }^{178} \mathrm{Ta}$ is the tantalum nucleus with the highest population, the obtained lifetime limits cover a higher range compared to the ${ }^{177,179} \mathrm{Ta}$ isotopes.

\section{F. ${ }^{179} \mathrm{Ta}$}

The ${ }^{179} \mathrm{Ta}$ nucleus, like ${ }^{179} \mathrm{~W}$, is populated in both ${ }^{134} \mathrm{Xe}$ and ${ }^{136} \mathrm{Xe}$ irradiations. According to the calculations, the cross sections are very close to each other in the two reactions. Since the results from the ${ }^{134} \mathrm{Xe}$ beam are more reliable, only these are given in Table III.

The case of the ${ }^{179} \mathrm{Ta}$ isotope is very similar to that of ${ }^{177} \mathrm{Ta}$. Only the transitions depopulating the low-lying $K=21 / 2$ isomer [21] at $1252 \mathrm{keV}$ are listed in Table III. It was assumed that $\sigma\left({ }^{179} \mathrm{~W}\right) / \sigma\left({ }^{179} \mathrm{Ta}\right)=\sigma\left({ }^{178} \mathrm{~W}\right) / \sigma\left({ }^{178} \mathrm{Ta}\right)$.

\section{G. ${ }^{175} \mathbf{H f}$}

An $\alpha$ particle has to be emitted by the compound nucleus to lead to the hafnium isotopes. The emission probability of an $\alpha$ particle is usually somewhat higher than that of a proton. Hence, based on the cross-section calculations, an $\alpha /$ proton emission ratio of 2 was adopted. Since the cross section of ${ }^{177} \mathrm{Hf}(K=37 / 2)$ was determined experimentally, the above assumption can be checked and it was found to be reasonable (see Sec. IV M).

It has to be noted that some transitions from the lower level of the ${ }^{175} \mathrm{Hf}$ nucleus are present in the spectra due to the $\beta$ decay of ${ }^{175} \mathrm{Ta}\left(T_{1 / 2}=10.5 \mathrm{~h}\right)$ probably produced as a consequence of the presence of ${ }^{44} \mathrm{Ca}$ and ${ }^{46} \mathrm{Ca}$ isotopes in the target. For this reason the lowest lying $81 \mathrm{keV} \gamma$-ray transition is not listed in Table III.

\section{H. ${ }^{176} \mathbf{H f}$}

The same cross-section assumption was adopted as for the ${ }^{175} \mathrm{Hf}$ nucleus. The lowest lying 88 and $202 \mathrm{keV} \gamma$ rays are not listed in Table III, since they can originate from the $\beta$ decay of ${ }^{176} \mathrm{Ta}\left(T_{1 / 2}=8.1 \mathrm{~h}\right)$, produced in the reaction on the ${ }^{44} \mathrm{Ca}$ - and ${ }^{46} \mathrm{Ca}$-contaminating isotopes in the target. 


\section{I. ${ }^{180} \mathrm{~W}$}

The ${ }^{180} \mathrm{~W}$ nucleus is the main product of the ${ }^{136} \mathrm{Xe}$ $+{ }^{48} \mathrm{Ca}$ reaction and according to the calculations its cross section is similar to that of ${ }^{178} \mathrm{~W}$ in the ${ }^{134} \mathrm{Xe}$ induced reaction. Based on these calculations it was assumed that $\sigma\left({ }^{180} \mathrm{~W}\right) / \sigma\left({ }^{47} \mathrm{Ca}\right){ }^{136}$ Xe-beam $=\sigma\left({ }^{178} \mathrm{~W}\right) / \sigma\left({ }^{47} \mathrm{Ca}\right){ }^{134}$ Xe-beam $\cdot$ The lifetime limits determined are in the same range as the values obtained for the other tungsten isotopes.

\section{J. ${ }^{181} \mathrm{~W}$}

It was assumed that the production cross section is equal to that of the production of ${ }^{179} \mathrm{~W}$ with the ${ }^{134} \mathrm{Xe}$ beam. The obtained lifetime limits are in the same range as for all the other tungsten isotopes.

\section{K. ${ }^{180} \mathrm{Ta}$}

The cross-section ratio of $\sigma\left({ }^{180} \mathrm{Ta}\right) / \sigma\left({ }^{47} \mathrm{Ca}\right){ }^{136}$ Xe-beam $=\sigma\left({ }^{178} \mathrm{Ta}\right) / \sigma\left({ }^{47} \mathrm{Ca}\right){ }^{134}$ Xe-beam was considered.

\section{L. ${ }^{181}$ Ta}

It was assumed that $\sigma\left({ }^{181} \mathrm{Ta}\right) / \sigma\left({ }^{47} \mathrm{Ca}\right){ }^{136} \mathrm{Xe}$-beam $=\sigma\left({ }^{179} \mathrm{Ta}\right) / \sigma\left({ }^{47} \mathrm{Ca}\right){ }^{134}$ Xe-beam. The $152-$ and $136-\mathrm{keV}$ transitions of ${ }^{181} \mathrm{Ta}$ are present in the spectra which are sensitive to the long half-lives, due to the $\beta$ decay of ${ }^{181} \mathrm{~W}$ which has a half-life of $121 \mathrm{~d}$.

\section{M. ${ }^{177} \mathbf{H f}$}

As stated in Sec. IV G we assumed, taking the mean value from the calculations, that the emission of an $\alpha$ particle is two times more probable than the emission of a proton. Since the cross section of the $K=37 / 2, T_{1 / 2}=51$ min state in ${ }^{177} \mathrm{Hf}$ has been determined relative to ${ }^{47} \mathrm{Ca}+{ }^{47} \mathrm{~K}$, this forms a check regarding the cross-section assumptions. From the experiment $\sigma\left({ }^{47} \mathrm{Ca}\right) /\left(\sigma\left({ }^{177} \mathrm{Hf}, K=37 / 2\right) \approx 130\right.$. According to our adopted assumptions, i.e., the population of the (HI, $4 n)$ channel is similar for the two beams, and the ratio of the $\alpha /$ proton evaporation probability is 2 , the ratio $\sigma\left({ }^{47} \mathrm{Ca}\right) /\left(\sigma\left({ }^{177} \mathrm{Hf}, K=37 / 2\right)\right.$ should be 165 if the isomer population were $30 \%$ of the population of the whole nucleus. The good agreement provides some support for the validity of the assumptions.

Although several $\gamma$-ray transitions have been checked for evidence of new isomers in ${ }^{177} \mathrm{Hf}$, only those populating the $51 \mathrm{~min}$ isomer [13] are listed in the table. It is worth mentioning that the spectra contain some low-lying ${ }^{177} \mathrm{Hf}$ transitions, coming from the $\beta$ decay of ${ }^{177} \mathrm{Ta}$.

\section{N. ${ }^{178} \mathbf{H f}$}

In the case of ${ }^{178} \mathrm{Hf}$, both the low-lying bands and that built on the $31 \mathrm{yr}$ isomer [32] have been checked. The recorded spectra contain some low-lying ${ }^{178} \mathrm{Hf}$ transitions, originating from the $\beta$ decay of ${ }^{178} \mathrm{Ta}$.

\section{DISCUSSION}

In our reactions, the nucleus ${ }^{178} \mathrm{~W}$ is one of the most strongly populated, and it is of particular interest with regard to possible long-lived isomers. Predictions for new high- $K$ states near the yrast line have been made by Jain et al. [3]. In a recent work by Purry et al. [6] many of the predicted isomeric levels were found, with half-lives up to $220 \mathrm{~ns}$. However, the experiment was not sensitive to very long-lived isomers, and indeed several of the predicted high- $K$ isomers were not found. These levels are predicted to have $K=26-30$ and the bandheads are thought to have excitation energies between 7 and $8 \mathrm{MeV}$. According to CASCADE calculations the states with spin higher than 30 should take about $50 \%$ of the total cross section. Since these isomers are predicted to be close to the yrast line, they would be expected to be populated in the present experiment. Assuming that only $1 \%$ of the intensity in this spin region passes through such isomeric levels: $F(\tau)=(F(\tau))_{\text {tab. } 3} /(100 \times 0.5 \times 0.01)$, one can conclude that the mean life of these isomers, if they exist, must be less than $0.7 \mathrm{~h}$ or more than $170 \mathrm{~d}$. A recent in-beam measurement [11] has identified $K=29,30$, and 34 bandheads, all with lifetimes less than $1 \mathrm{~ns}$.

Similar considerations could apply to the other tungsten isotopes. However, the nuclei populated after the evaporation of charged particles, namely the tantalum and hafnium nuclei, have much smaller cross sections, and usually their values are not known accurately. For these reasons, half-life limits usually can be obtained only if we assume $f_{\text {ex }}>0.1$.

In ${ }^{180} \mathrm{Ta}$ a long-lived $K^{\pi}=22^{-}$isomer was predicted but not observed [4]. A parallel work by Saitoh et al. [7] also missed the identification of this state either because of the low spin input of the ${ }^{11} \mathrm{~B}$ induced reaction or the long lifetime. However, in our heavy-ion induced reaction the cross section for states with spin $\geqslant 22$ is around $65 \%$ compared to the total production cross section of the nucleus. Assuming that $50 \%$ of the intensity in this spin region passes through the predicted isomeric level: $F(\tau)=(F(\tau))_{\mathrm{tab} .3} /(10 \times 0.65$ $\times 0.5)$, one can conclude that the mean life of this isomer must be less than $1.2 \mathrm{~h}$ or more than $11 \mathrm{~d}$. We note that $K=22$ and 24 isomers have since been discovered by Wheldon et al. [33], with microsecond half-lives.

In ${ }^{177} \mathrm{Ta}$ a long-lived $K^{\pi}=67 / 2^{-}$isomer is predicted [8]. The calculations indicate a cross section of about $20 \%$ for this spin. However, it is difficult to give an accurate estimate since this high spin is relatively close to the highest spin input. Using the calculated value we conclude that the mean life of the predicted $67 / 2^{-}$nine-quasiparticle state cannot be between 1 and $9 \mathrm{~d}$. The identification of this state remains an experimental challenge.

The lower limits of the lifetime are summarized in Table IV. In each case it was assumed that any new isomer formed would decay through the already known isomeric states or the upper part of the corresponding yrast band, and that the isomer population cross section compared with the total one of the nucleus is either $1 \%, 10 \%$, or $30 \%$.

The nonobservation of an isomer does not necessarily mean that the searched-for multiquasiparticle state is not isomeric. It indicates only that the respective multiquasiparticle state does not have a lifetime in the range of the sensitivity of the experiment and/or it was not populated with sufficient cross section. 
TABLE IV. The deduced lower mean lives considering isomer population of $1 \%, 10 \%$, and $30 \%$ compared to the production of the nucleus. For further explanation see the text.

\begin{tabular}{|c|c|c|c|c|}
\hline \multirow[b]{2}{*}{ Nucleus } & \multirow[b]{2}{*}{$E_{\gamma}(\mathrm{keV})\left(\mathrm{I}_{i}^{\pi} \rightarrow \mathrm{I}_{f}^{\pi}\right)$} & \multicolumn{3}{|c|}{$\tau$ limit (h) } \\
\hline & & $f_{\mathrm{ex}}=0.01$ & $f_{\mathrm{ex}}=0.1$ & $f_{\mathrm{ex}}=0.3$ \\
\hline${ }^{177} \mathrm{~W}$ & $256\left(11 / 2^{-} \rightarrow 7 / 2^{-}\right)$ & 2700 & 27000 & 83000 \\
\hline${ }^{178} \mathrm{~W}$ & $237\left(4^{+} \rightarrow 2^{+}\right)$ & 8500 & 85000 & 260000 \\
\hline${ }^{179} \mathrm{~W}$ & $359\left(15 / 2^{-} \rightarrow 11 / 2^{-}\right)$ & 3700 & 32000 & 94000 \\
\hline${ }^{180} \mathrm{~W}$ & $234\left(4^{+} \rightarrow 2^{+}\right)$ & 2500 & 25000 & 76000 \\
\hline${ }^{181} \mathrm{~W}$ & $348\left(17 / 2^{+} \rightarrow 13 / 2^{+}\right)$ & 610 & 6800 & 21000 \\
\hline${ }^{177} \mathrm{Ta}$ & $312\left(21 / 2^{-} \rightarrow 19 / 2^{-}\right)$ & - & - & 390 \\
\hline${ }^{178} \mathrm{Ta}$ & $219\left(9^{-} \rightarrow 8^{-}\right)$ & - & 250 & 940 \\
\hline${ }^{179} \mathrm{Ta}$ & $232\left(21 / 2^{-} \rightarrow 19 / 2^{-}\right)$ & - & - & 94 \\
\hline${ }^{180} \mathrm{Ta}$ & $269\left(13^{-} \rightarrow 12^{-}\right)$ & - & 23 & 120 \\
\hline${ }^{181} \mathrm{Ta}$ & $179\left(13 / 2^{-} \rightarrow 11 / 2^{-}\right)$ & - & - & 51 \\
\hline${ }^{175} \mathrm{Hf}$ & $127\left(11 / 2^{-} \rightarrow 9 / 2^{-}\right)$ & - & 150 & 640 \\
\hline${ }^{176} \mathrm{Hf}$ & $307\left(6^{+} \rightarrow 4^{+}\right)$ & - & 330 & 1200 \\
\hline${ }^{177} \mathrm{Hf}$ & $366\left(39 / 2^{+} \rightarrow 37 / 2^{-}\right)$ & - & 270 & 860 \\
\hline${ }^{178} \mathrm{Hf}$ & $377\left(18^{+} \rightarrow 17^{+}\right)$ & - & 69 & 240 \\
\hline
\end{tabular}

Since the search for multiquasiparticle isomers in the $A$ $\approx 180$ region will continue, the results of the present experiment, i.e., the extracted lifetime limits, can be used to plan further investigations.

In summary, limits have been established for long-lived high- $K$ isomer production in tungsten, tantalum, and hafnium isotopes. While no new isomers have been established in the present work, the limits observed may be helpful in planning future experiments. The predicted $K^{\pi}=67 / 2^{-}$ninequasiparticle isomer in ${ }^{177} \mathrm{Ta}$ remains of particular interest.
[1] R. G. Helmer and C. W. Reich, Nucl. Phys. A211, 1 (1973).

[2] S. Åberg, Nucl. Phys. A306, 89 (1978).

[3] K. Jain, O. Burglin, G. D. Dracoulis, B. Fabricius, N. Rowley, and P. M. Walker, Nucl. Phys. A591, 61 (1995).

[4] G. D. Dracoulis, A. P. Byrne, S. M. Mullins, T. Kibédi, F. G. Kondev, and P. M. Davidson, Phys. Rev. C 58, 1837 (1998).

[5] P. M. Walker and G. D. Dracoulis, Nature (London) 399, 35 (1999).

[6] C. S. Purry, P. M. Walker, G. D. Dracoulis, T. Kibédi, F. Kondev, S. Bayer, A. M. Bruce, A. P. Byrne, W. Gelletly, P. H. Regan, C. Thwaites, O. Burglin, and N. Rowley, Nucl. Phys. A632, 229 (1998).

[7] T. R. Saitoh, N. Hashimoto, G. Sletten, R. A. Bark, S. Tormanen, M. Bergstrom, K. Furuno, K. Furutaka, G. B. Hagemann, T. Hayakawa, T. Komatsubara, A. Maj, S. Mitarai, M. Oshima, J. Sampson, T. Shizuma, and P. G. Varmette, Nucl. Phys. A660, 121 (1999).

[8] M. Dasgupta, P. M. Walker, G. D. Dracoulis, A. P. Byrne, P. H. Regan, T. Kibédi, G. J. Lane, and K. C. Yeung, Phys. Lett. B 328, 16 (1994).

[9] M. Dasgupta, G. D. Dracoulis, P. M. Walker, A. P. Byrne, T. Kibédi, F. G. Kondev, G. J. Lane, and P. H. Regan, Phys. Rev. C 61, 044321 (2000).

[10] G. D. Dracoulis, F. G. Kondev, and P. M. Walker, Phys. Lett. B 419, 7 (1998).

[11] D. M. Cullen, S. L. King, A. T. Reed, J. A. Sampson, P. M. Walker, C. Wheldon, F. Xu, G. D. Dracoulis, I.-Y. Lee, A. O.
Macchiavelli, R. W. MacLeod, A. N. Wilson, and C. Barton, Phys. Rev. C 60, 064301 (1999).

[12] S. Frauendorf, K. Neergård, J. A. Sheikh, and P. M. Walker, Phys. Rev. C 61, 064324 (2000).

[13] S. M. Mullins, A. P. Byrne, G. D. Dracoulis, T. R. McGoram, and W. A. Seale, Phys. Rev. C 58, 831 (1998).

[14] C. Wheldon, R. D'Alarcao, P. Chowdbury, P. M. Walker, E. Seabury, I. Ahmad, M. P. Carpenter, D. M. Cullen, G. Hackman, R. V. F. Ranssens, T. L. Khoo, D. Nisius, C. J. Pearson, and P. Reiter, Phys. Lett. B 425, 239 (1998).

[15] Zs. Podolyák, P. H. Regan, M. Pfützner, J. Gerl, M. Hellström, M. Caamaño, P. Mayet, M. Mineva, M. Sawicka, and Ch. Schlegel, for the GSI ISOMER Collaboration, in Proceedings of the International Conference on Fission and Neutron-rich Nuclei, St. Andrews, Scotland 1999 (World Scientific, Singapore), p. 156.

[16] H. R. Andrews et al., Report No. AECL-8329, 1984 (unpublished).

[17] Table of Isotopes, edited by R. B. Firestone and V. S. Shirley, 8th ed. (Wiley, New York, 1996).

[18] T. E. Ward and P. E. Haustein, Phys. Rev. Lett. 27, 685 (1971).

[19] A. Gavron, Phys. Rev. C 21, 230 (1980).

[20] F. Puhlhofer, Nucl. Phys. A280, 267 (1977).

[21] F. G. Kondev, G. D. Dracoulis, A. P. Byrne, T. Kibédi, and S. Bayer, Nucl. Phys. A617, 91 (1997).

[22] T. Shizuma, G. Sletten, R. A. Bark, I. G. Bearden, S. Leoni, 
M. Mattiuzzi, S. Mitarai, S. W. Odegard, S. Skoda, K. Strahle, J. Wrzesinski, and Y. R. Shimizu, Nucl. Phys. A626, 760 (1997)

[23] P. M. Walker, G. D. Dracoulis, A. P. Byrne, B. Fabricius, T. Kibédi, A. E. Stuchbery, and N. Rowley, Nucl. Phys. A568, 397 (1994).

[24] F. G. Kondev, G. D. Dracoulis, A. P. Byrne, and T. Kibédi, Nucl. Phys. A632, 473 (1998).

[25] A. O. Macchiavelli and E. Browne, Nucl. Data Sheets 69, 903 (1993).

[26] E. Browne and J. Huo, Nucl. Data Sheets 84, 337 (1998).

[27] E. Browne, Nucl. Data Sheets 71, 81 (1994).
[28] R. B. Firestone, Nucl. Data Sheets 62, 101 (1991).

[29] G. D. Dracoulis, F. G. Kondev, A. P. Byrne, T. Kibédi, S. Bayer, P. M. Davidson, P. M. Walker, C. Purry, and C. J. Pearson, Phys. Rev. C 53, 1205 (1996).

[30] G. D. Dracoulis, S. M. Mullins, A. P. Byrne, F. G. Kondev, T. Kibédi, S. Bayer, G. J. Lane, T. R. McGoram, and P. M. Davidson, Phys. Rev. C 58, 1444 (1998).

[31] E. Browne, Nucl. Data Sheets 72, 221 (1994).

[32] S. M. Mullins, G. D. Dracoulis, A. P. Byrne, T. R. McGoram, S. Bayer, W. A. Seale, and F. G. Kondev, Phys. Lett. B 393, 279 (1997).

[33] C. Wheldon et al. (unpublished). 